\title{
Can oral surgery be performed safely when COVID-19 status is unknown?
}

\section{Adam Jones*1 $^{* 1}$ and Gavin Wilson ${ }^{1}$}

\section{A commentary on}

\section{Alterman M, Nassar M, Rushinek H, Cohen A, Shapira L, Casap N.}

The efficacy of a protective protocol for oral and maxillofacial surgery procedures in a COVID-19 pandemic area-results from 1471 patients. Clin Oral Investig 2021; DOI: 10.1007/s00784-021-03809-8.

\begin{abstract}
Design Cross-sectional study/special report.

Study population This paper presents the early experience of the oral and maxillofacial surgery department at Hadassah University Medical Centre in Jerusalem during the first wave of the COVID-19 pandemic in 2020. The study involved both medical staff and patients.

Data analysis A retrospective analysis of an eight-week period (February-April 2020) collated 1,471 patient records and examined diagnoses, procedures performed and COVID-19 status of patients and staff. Any attempts made to access routine dental care before presentation in secondary care were recorded.
\end{abstract}

Results In the study period, one member of staff was confirmed as COVID-19-positive. Sixty-three patients had formal COVID-19 tests; all were negative. Forty-three patients were admitted for drainage of odontogenic fascial space infections; $53 \%$ reported delayed or failed attempts to access dental care before their infection. Additionally, the authors describe a screening process, personal protective equipment (PPE) allocation and staff/patient testing protocols employed in their surgical unit throughout this period.

Conclusions The authors suggest a series of triage and screening measures to limit the risk of unknowingly exposing clinical staff to the COVID-19 virus and offer advice on safely delaying non-emergency treatment where necessary. Recommendations for use of PPE for aerosol and non-aerosol generating procedures are made, but it is important to recognise that the efficacy of these measures cannot be determined by the methodology employed. This paper demonstrates an early example of complications developing from absent or delayed routine dental services resulting from lockdowns. This 'excess morbidity' is likely to have an impact on healthcare services as the pandemic recovery unfolds and services begin to return to normal.

\section{Commentary}

This paper presents the experience of an oral and maxillofacial surgery department in a pandemic region during the first wave of the COVID-19 pandemic. $^{1}$ Despite limitations and significant methodological flaws, it offers insight into efforts to mitigate the

\section{GRADE rating}

\section{Practice points}

- The evidence base for aerosol transmission specific to dentistry is limited at present but is continually improving. Oral surgery teams, and general dental teams, must stay informed of local and national guidance.

- Demand for oral surgery services is likely to be high with a backlog of care, following disruption to business-as-usual operations. Patients should be risk-assessed and prioritised as per clinical need, ensuring efficient and effective use of available resources.

risk of unknown transmission of COVID-19 and precautionary measures taken when performing oral surgery procedures from a period when there was little evidence for recommendations.

During the COVID-19 pandemic, most countries have categorised oral and maxillofacial surgical interventions as 'highrisk aerosol generating procedures' ${ }^{2}$ This reflects the routine manipulation of tissues with high viral loads within the oral cavity and oropharynx during procedures, and the routine use of surgical drills or cautery which may cause aerosolisation of fluids.

Transmission of COVID-19 has been reported to be more likely in aerosolised form, ${ }^{3}$ and the efforts to protect staff and patients described by this paper focused on limiting the possibility of encountering infected patients unknowingly. The authors describe a 'quadruple screening protocol' which involved: several symptom-screening opportunities by telephone and in person; patient temperature screening on attendance; protective personal equipment (PPE) for patients and staff; pre-procedural mouthwashes; and a designated negative-pressure room assigned for positive patients meeting criteria for urgent surgery. ${ }^{1}$

It is important to recognise that the effectiveness of the measures described by this paper cannot be determined by the methodology employed. Important information, such as the infection prevalence in the population, was not available and the few COVID-19 tests performed were negative (63 patients were tested in total). One member of the surgical team tested positive during the study period, which was attributed to exposure in a non-clinical setting, but no details of this case were provided. It is worth noting that infection was not passed to other members of the team, which the authors attribute to their PPE protocols and early detection by regular staff testing.

Aerosolised transmission of COVID-19 clearly presents an ongoing risk in dental practice, where high-speed air rotors, which aerosolise saliva, are frequently used. Aerosol is produced by most dental procedures and patient interactions, including speaking, coughing and sneezing. ${ }^{4}$ The transmissibility of particles is greater with smaller particle sizes. The size variances can be described as: splatter $(>100 \mu \mathrm{m})$, droplets $(>5 \mu \mathrm{m})$ or droplet nuclei $(<5 \mu \mathrm{m}){ }^{5}$ The former are larger, heavier particles that, according to a National Services Scotland technical report, will settle up to ten minutes following cessation of production. ${ }^{6}$ 
This is of great relevance to the operational practice of oral surgery departments with regards to fallow time and patient throughput.

The oropharynx is a known source of SARS-CoV-2 in an infected individual. ${ }^{7}$ However, the risk of transmission, specifically in a dental setting, is dependent on the quantity of virus present in the oral cavity. The quantity and viability of SARS-CoV-2 in the aerosol produced by dental procedures is of great significance. Nonetheless, there is a potential risk of transmission and it is important that proportionate mitigations are implemented. Mitigations can include a combination of environmental and procedural factors including ventilation, PPE, high-volume suction, rubber dam and a fallow period. Detailed summaries on the various mitigations available can be found in a publication by the Scottish Clinical Effectiveness Programme titled Mitigation of aerosol generating procedures in dentistry. ${ }^{5}$

A recent study by Allison et al. aimed to assess the level of aerosol produced from three common dental procedures: ultrasonic scaling, high-speed air turbine and 3-in-1 syringe. ${ }^{8}$ Unfortunately, these procedures are uncommon in oral surgery and therefore of limited use in this context. However, a commonly used instrument within the speciality of oral surgery is the oral surgical handpiece. This is different to a traditional air turbine handpiece and is commonly electrically operated at much slower revolutions per minute (RPM) (40,000 RPM). It is therefore likely that an oral surgical handpiece produces splatter and droplets, which can be mitigated with standard infection control precautions, widely adopted in dentistry before the emergence of COVID-19. ${ }^{9}$

Excess mortality and morbidity are expected consequences during a pandemic which has resulted in stretched healthcare systems and national lockdowns which have limited or delayed access to care. ${ }^{10} \mathrm{~A}$ final point of interest reported by the authors was a high proportion of patients (53\%) admitted for drainage of odontogenic fascial space infections having experienced delayed or failed access to dental care before their infection developing. These examples of escalation to more complex treatment or emergency intervention to remedy preventable complications may become more apparent as services recover and societies return to normal. In the UK, it is estimated that ten million dental appointments have been delayed due to COVID-19. ${ }^{11}$ In the wider healthcare setting, reduced attendances at emergency departments and fewer cancer diagnoses raise concerns of disease progressing untreated and poorer eventual outcomes. This secondary impact is important to consider as services are restored to pre-pandemic levels.

\section{Author affiliation \\ ${ }^{1}$ Department of Oral Surgery, Leeds Dental Institute, UK. ${ }^{*}$ Correspondence to: Adam Jones}

\section{References}

1. Alterman $M$, Nassar $M$, Rushinek $H$, Cohen A, Shapira L, Casap N. The efficacy of a protective protocol for oral and maxillofacial surgery procedures in a COVID-19 pandemic area - results from 1471 patients. Clin Oral Investig 2021; DOI: 10.1007/ s00784-021-03809-8.

2. Howard B E. High-risk aerosol-generating procedures in COVID-19: respiratory protective equipment considerations. Otolaryngol Head Neck Surg 2020; 163: 98-103.

3. Baraniuk C. Covid-19: What do we know about airborne transmission of SARSCoV-2? BMJ 2021; DOI: 10.1136/bmj.n1030.

4. Hinds W C. Aerosol Technology: Properties, Behaviour, and Measurement of Airborne Particles. 2nd ed: Hoboken: Wiley, 1999.

5. Scottish Dental Clinical Effectiveness Programme. Mitigation of Aerosol Generating Procedures in Dentistry. 2020. Available at https://www.sdcep.org.uk/wp-content/ uploads/2020/12/SDCEP-Mitigation-of-AGPs-Review-Methodology.pdf (accessed April 2021).

6. National Services Scotland. Ventilation, water and environmental cleaning in dental surgeries relating to COVID-19. 2020. Available at https://www.scottishdental. org/ventilation-water-and-environmental-cleaning-in-dental-surgeries-relating-tocovid-19/ (accessed April 2021).

7. To K K, Tsang O T, Yip C C et al. Consistent Detection of 2019 Novel Coronavirus in Saliva. Clin Infect Dis 2020; 71: 841-843.

8. Allison J R, Currie C C, Edwards D C et al. Evaluating aerosol and splatter following dental procedures: Addressing new challenges for oral health care and rehabilitation. J Oral Rehabil 2020; 48: 61-72.

9. Ishihama $\mathrm{K}$, lida $\mathrm{S}$, Koizumi $\mathrm{H}$ et al. High incidence of blood exposure due to imperceptible contaminated splatters during oral surgery. J Oral Maxillofac Surg 2008; 66: 704-710

10. Zylke J W, Bauchner H. Mortality and morbidity: the measure of a pandemic. JAMA 2020; 324: 458-459.

11. Evans S. More than 10 million dental appointments delayed due to COVID19. 2020. Available at https://dentistry.co.uk/2020/08/24/10-million-dentalappointments-delayed-due-covid/ (accessed April 2021).

Evidence-Based Dentistry (2021) 22, 74-75.

https://doi.org/ 10.1038/s41432-021-0176-0 